\title{
Use of cluster-graphs from spoligotyping data to study genotype similarities and a comparison of three indices to quantify recent tuberculosis transmission among culture positive cases in French Guiana during a eight year period
}

Vanina Guernier*1, Christophe Sola2,3,4, Karine Brudey2,5, JeanFrançois Guégan ${ }^{1}$ and Nalin Rastogi ${ }^{2}$

\begin{abstract}
Address: ${ }^{1}$ UMR 2724 IRD-CNRS, Génétique et Évolution des Maladies Infectieuses, Equipe Dynamique des Systèmes \& Maladies Infectieuses, 911 avenue Agropolis, BP 64501, 34394 Montpellier Cedex 05, France, ${ }^{2}$ Unité de la Tuberculose et des Mycobactéries, Institut Pasteur de Guadeloupe, BP 484, 97183 Abymes Cedex, Guadeloupe, ${ }^{3}$ Institut de Génétique et de Microbiologie UMR8621, CNRS, Université Paris-Sud 11, 91405 Orsay, France, ${ }^{4}$ Unité de Génétique Mycobactérienne, Institut Pasteur, 25 rue du Dr Roux, 75724 Paris Cedex 15, France and ${ }^{5}$ Centre Hospitalier Universitaire, Route de Chauvel, BP 465, 97139 Pointe-à-Pitre Cedex, Guadeloupe
\end{abstract}

Email: Vanina Guernier* - vanina.guernier@u707.jussieu.fr; Christophe Sola - csola@pasteur.fr; Karine Brudey - karystel@yahoo.fr; JeanFrançois Guégan - guegan@mpl.ird.fr; Nalin Rastogi - nrastogi@pasteur-guadeloupe.fr

* Corresponding author

Published: 14 April 2008

BMC Infectious Diseases 2008, 8:46 doi:10.1 186/147/-2334-8-46
Received: 6 November 2007

Accepted: 14 April 2008

This article is available from: http://www.biomedcentral.com/I47I-2334/8/46

(C) 2008 Guernier et al; licensee BioMed Central Ltd.

This is an Open Access article distributed under the terms of the Creative Commons Attribution License (http://creativecommons.org/licenses/by/2.0), which permits unrestricted use, distribution, and reproduction in any medium, provided the original work is properly cited.

\begin{abstract}
Background: French Guiana has the highest tuberculosis (TB) burden among all French departments, with a strong increase in the TB incidence over the last few years. It is now uncertain how best to explain this incidence. The objective of this study was to compare three different methods evaluating the extent of recent TB transmission in French Guiana.

Methods: We conducted a population-based molecular epidemiology study of tuberculosis in French Guiana based on culture-positive TB strains (1996 to 2003, $n=344$ ) to define molecular relatedness between isolates, i.e. potential transmission events. Phylogenetic relationships were inferred by comparing two methods: a "clustergraph" method based on spoligotyping results, and a minimum spanning tree method based on both spoligotyping and variable number of tandem DNA repeats (VNTR). Furthermore, three indices attempting to reflect the extent of recent TB transmission (RTIn, RTIn-I and TMI) were compared.

Results: Molecular analyses showed a total amount of 120 different spoligotyping patterns and 273 clinical isolates $(79.4 \%)$ that were grouped in 49 clusters. The comparison of spoligotypes from French Guiana with an international spoligotype database (SpolDB4) showed that the majority of isolates belonged to major clades of $M$. tuberculosis (Haarlem, 22.6\%; Latin American-Mediterranean, 23.3\%; and T, 32.6\%). Indices designed to quantify transmission of tuberculosis gave the following values: $\mathrm{RTIn}=0.794, \mathrm{RTIn}-\mathrm{I}=0.65 \mathrm{I}$, and TMI $=0.146$.

Conclusion: Our data showed a high number of Mycobacterium tuberculosis clusters, suggesting a high level of recent TB transmission, nonetheless an estimation of transmission rate taking into account cluster size and mutation rate of genetic markers showed a low ongoing transmission rate (14.6\%). Our results indicate an endemic mode of TB transmission in French Guiana, with both resurgence of old spatially restricted genotypes, and a significant importation of new TB genotypes by migration of TB infected persons from neighgouring highincidence countries.
\end{abstract}




\section{Background}

The resurgence of human tuberculosis (TB) in many industrialized countries in the late 1980s and early 1990s [1] has focused critical attention on a disease that was thought to be under control, and as such, has been neglected for too long. Effective prevention and control of Mycobacterium tuberculosis, the causative agent of TB, must be based on a clear understanding of how the disease is transmitted, how infection becomes established, and how infection progresses to clinical disease. This has motivated investigations on TB transmission among persons at higher risk, such as the homeless [2,3] or HIV-infected persons [4,5]. Furthermore, the improvement of molecular techniques to characterize Mycobacterium strains, especially M. tuberculosis, and the concomitant accumulation of epidemiological data over large spatial scales has increased the possibility to trace transmission routes of Mycobacterium outbreaks (e.g. M. leprae in [6]). The identification of $M$. tuberculosis strains can be used to identify patients which have some apparently identical genotypes, thus potentially associated to a same source of contamination [7] and designated as "clustered cases» [8,9]. The existence of genetic clusters is thought to be indicative of recent TB transmission $[10,11]$, in opposition to cases arising either from imports or from reactivation of latent infection. Featuring epidemiological links between patients allow inferences about the level of ongoing TB transmission, thus the level of epidemicity/endemicity of the infection in a defined geographical area, and underline some potential risk factors associated to the disease [12]. For example, when an unusual number of TB cases occurs over time, it is possible to do early molecular analyses to determine whether this cluster of cases represents temporal coincidence, or a genetic cluster due to a local chain of transmission. If fingerprinting demonstrates different strains, the cases are not due to transmission, and there is no need for further epidemiological evaluation.

The underlined hypothesis of the use of molecular information for the evaluation of ongoing transmission is that mutations are slower than transmission rate [13], which is not true for all genetic markers. A large number of molecular methods are available for the characterization of infra-specific $M$. tuberculosis strains. Nonetheless the choice of a method strongly affects the results, as low mutation rates tend to less discriminate the genetic differences, whereas high mutation rates tend to increase the number of clusters considered [14]. This article investigates the genotypic diversity of $M$. tuberculosis strains isolated in French Guiana over the 1996-2003 period, characterized by two molecular methods, and attempts to determine potential transmission between infected persons. We used a recent method proposed by Tanaka \& Francis [14] to visualise molecular epidemiological data, i.e. cluster-graphs, which also provides a framework for evaluating ongoing transmission. We also compared the method used to previous indices attempting to reflect the extent of recent $\mathrm{TB}$ transmission.

\section{Methods \\ Clinical isolates}

French Guiana is a French overseas department of 32,432 sq mi. It is located on the north-east of South America, between Suriname and Brazil. The region has the highest TB rates among all French departments, with an average annual incidence of $63.1 \mathrm{~TB}$ cases per 100,000 population estimated during the period 1996-2003 [15]. Among all TB cases identified from January 1, 1996 to December 31, 2003, 345 were confirmed by isolation and cell culture of M. tuberculosis by the Mycobacterium Laboratory of the Institut Pasteur de la Guyane (IPG). A total of 344 clinical isolates of $M$. tuberculosis were isolated from patients with culture-positive $\mathrm{TB}$, representing 342 patients (two patients only were contaminated twice, by two different strains). The three missing cases $(0,86 \%)$ correspond to contaminated cultures. After a prior explanation of the recommended investigations for the diagnostic purposes by a physician, patients were asked to provide oral consent and the pathological specimens were obtained from suspected tuberculosis patients residing in French Guiana. The cultures were performed using Löwenstein-Jensen slants at $37^{\circ} \mathrm{C}$ at the Pasteur Institute of French Guiana. Positive cultures (where no identification was attached) were sent to the Pasteur Institute of Guadeloupe which has been serving as a regional reference laboratory for tuberculosis and mycobacterial for the Caribbean since 1994. The cultures were duly identified as M. tuberculosis complex using classical biochemical tests and the AccuProbe test (GenProbe Inc., San Diego, CA) and subjected to drug-susceptibility testing using the proportional method. The bacteriological results were communicated to the physicians and/or hospital services responsible for patient care and treatment, through the Pasteur Institute of French Guiana. DNA-based molecular typing was also performed (see below).

\section{Molecular typing}

Molecular typing was performed using spoligotyping and VNTRs analyses. It was previously suggested that a combined spoligotyping-5 VNTR loci genotyping scheme may have a discriminatory power close to that of IS6110 RFLP, the historical "golden standard" in tuberculosis genotyping [16]. Hence, for ease and resources reasons, we choosed this scheme, which was less expensive in time and money than the combined spoligotyping-12 VNTR loci scheme, i.e. MIRUs [17]. Recent improvement in VNTR typing now recommended a new "universal" 15 or even 24 VNTR loci genotyping scheme for M. tuberculosis [18]. 
The bacterial DNA was prepared by the classical cetyl-trimethyl ammonium bromide method (CTAB) [19] and used for spoligotyping (spacer oligonucleotide typing) and Variable Number of DNA Tandem Repeats (VNTRs) analyses. Spoligotyping was performed using a homemade membrane with 43 covalently bound oligonucleotides and PCR was achieved using primers designated DRa and DRb, with DRa biotinylated 5' to amplify the whole DR region as described previously [20]. As spoligotyping used alone is known to overestimate the number of epidemiological links (depending on the settings, but by around 30\%), it was suggested to be used in association with another rapid fingerprinting technique [21]. Hence, the isolates clustered by spoligotyping (and only those first-clustered isolates) were further subtyped using VNTRs as described previously [22] in order to provide a second independent indicator of clonality. The number of copies for each exact tandem repeat (ETR) was documented as a five-digit numbers representing allele profiles ETR-A to ETR-E [23].

\section{Strains matching}

Spoligotype patterns are designated as 43-character-long strings consisting of black and white squares, indicating respectively the presence or the absence of an individual spacer. The totality of the 344 isolates was entered in this binary format as Excel (Microsoft, Cupertino, CA) spreadsheets. The spoligotype designations (shared types or ST, defined as an identical spoligotype found in $\geq 2$ individual patient isolates) were attributed by comparing the patterns obtained with those included in an international spoligotyping database held at the Pasteur Institute of Guadeloupe, designated as spolDB4 [24]. At the time of the matching analysis, the updated spolDB4 version contained 31,642 patterns distributed into 2,393 shared types in 114 countries (an online version of this database is now available at http://www.pasteur-guadeloupe.fr:8081/ SITVITDemo/). Patterns referenced only once are designated as true orphans (in opposition to pseudo-orphans, i.e. isolates found to be unique in this study but for whom a counterpart exists in the database) and are not labelled with an ST number. Major phylogenetic clades were assigned to STs according to signatures provided in SpolDB4 (the reader is referred to the original paper [24] for a detailed description). These included specific signatures for various $M$. tuberculosis sub-species (M. bovis, $M$. microti, M. caprae, M. pinipedii, M. africanum), as well as rules defining major lineages/sub-lineages for M. tuberculosis stricto sensu. The latter included the Central-Asian (CAS) clade and 2 sub-lineages; the East-African-Indian (EAI) clade and 9 sub-lineages; the Haarlem $(\mathrm{H})$ clade and 3 sub-lineages; the Latin-American-Mediterranean (LAM) clade and 12 presumed sub-lineages; the "Manu" family and 3 sub-lineages; the S clade; the IS6110-low banding X clade and 3 sub-lineages; and an ill-defined T clade with 5 sub-lineages. The T clade corresponds to Principal Genetic Groups (PGG) 2 and 3, and remains defined by default (no specific signature sequences). M. tuberculosis families are phylogeographically-specific, as generally indicated by their names.

\section{Phylogeography and cluster-graphs}

Data were analysed by grouping isolates into clusters of identical genotypes and by organizing all genotypes present in the overall sample according to genetic relationships, i.e. by constructing a phylogeny. We used and compared two methods for inferring potential phylogenetic relationships: (1) a classification based on spoligotyping results, which we call a "cluster-graph" and which is based on parsimony principles, and (2) a minimum spanning tree (MST) method which is based on a spoligotyping and VNTR combined distance matrix, computed using the BioNumerics software (Applied Maths, Sint Marten-Latems, Belgium).

A cluster-graph is a new graphic illustration developed by Tanaka and Francis [14] for representing and analysing clonal pathogenic genotypes. The graphic tree is drawn with genotypes at internal nodes, edges reflecting direct evolutionary relationships between them, and the size of the circle at the nodes being proportional to cluster sizes. The first step is to form clusters of $g$ distinct genotypes $(g$ nodes), then to connect all vertices whose genotypes are separated by a single mutation step [14]. In our study, connections between genotypes separated by more than one mutation step were visualized within concentric successive circles which illustrated the increased number of mutation events gained from inside to outside. The Figure 1 illustrates the difference between cluster-graph published by Tanaka and Francis, and the adaptation that we made in the present exercise. The "BioLayout" software was used to draw the networks (cluster-graph) representing genotype similarities, similarly as what can be done for proteins considering their amino-acid content $[25,26]$.

The cluster-graph method could not be applied to the combined spoligotyping-VNTR data, as the manual inference of tree edges between genotypes needs to be supported by the number of mutations events between different genotypes. The VNTR analyses giving occurrence of ETR-A to E do not give any information about the genetic distance between different VNTR patterns. VNTR analyses were included on the construction of the minimum spanning tree method, in order to provide further discrimination between strains for isolates with identical spoligotyping results, Distance matrices are calculated and the obtained groupings can be used to build a dendrogram. 
A)
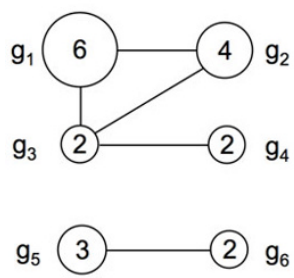

$$
\begin{aligned}
& n=6+4+2+2+3+2=19 \\
& g=6 \\
& v_{1}=(4-1)+(2-1)=4
\end{aligned}
$$

\section{Figure I}

Cluster-graph representing a sample of 19 TB cases with six distinct genotypes. Each node represents a cluster of genotypically identical isolates, and the sizes of clusters are indicated inside the nodes. (A) Illustration from Tanaka and Francis (2005). The arrow indicates the sense of evolution through mutation events. (B) Our own representation. Dotted circles indicates mutation steps, with one mutation step between $\mathrm{gl}$ and $\mathrm{g} 3$, but two between $\mathrm{gl}$ and $\mathrm{g} 4$, for instance.

\section{Estimation of transmission}

Three indices of outbreak severity were calculated and compared. The two first ones are referred to as recent transmission indices (RTI) because they are intended to reflect the extent of recent transmission of tuberculosis [14]. The first index is $\operatorname{RTI}_{n}=n_{c} / n$ [10], where $n$ is the total number of cases in the sample and $n_{c}$ is the total number of cases in cluster (size two or greater). The second one is $\mathrm{RTI}_{n-1}=\left(n_{c}-c\right) / n[11]$, where $c$ is the number of genotypes represented by at least two cases; i.e. one case per cluster, which is supposed to be due to reactivation of a previous TB case, is taken off. These have been referred to as the " $n$ method" and the "n-1 method" respectively $[27,28]$. The underlined idea is that a more genetically homogeneous data set would represent a more severe extent of disease transmission, i.e. an "outbreak". The third index is the transmission mutation index (TMI) [14].

$$
T M I=\tilde{\mu} \frac{\left(n-g+v_{1}\right)}{v_{1}}
$$

where $\tilde{\mu}$ is an independent estimate of the mutation rate of the genetic marker, and $v_{1}$ is the number of single-step mutation events inferred from the data [14]. We used a mutation rate for spoligotypes of 0.039 events per year computed from previous estimates in the literature $[14,29]$. All three indices are expressed as a ratio from 0 to 1 , which can also be expressed in percentage. It must be interpreted in terms of "percent of isolates in cluster", which is indicative of the rate of recent TB transmission. Thus, a result of 0.5 indicates that $50 \%$ of the isolates of a data set were probably related to a recent transmission event.

\section{Results \\ Molecular typing results}

The spoligotyping was performed as a first-line screening method on $344 \mathrm{M}$. tuberculosis clinical isolates from patients living in French Guiana, followed by VNTR performed on 273 clustered isolates. Among the 344 typed isolates, spoligotyping generated 49 clustered patterns totalling 273 isolates (with 2 to 48 patients per cluster), 25 true orphan patterns, and 46 pseudo-orphans, i.e. patterns already found in SpolDB4 but present as single in this study, with a total amount of 120 different patterns. Analysis of the frequency of major spoligotypes with SpolDB4 allowed a differentiation between ubiquitous types present in all the continents and types more specific to French Guiana. Several types were endemic in French Guiana (i.e. ST 66, 76, 94, 1084, 1340, 1526 and 25 orphan isolates), representing $25.8 \%$ of the shared-types, and $12.2 \%$ of the total isolates. Ubiquitous types represent $40.8 \%$ of the 120 individual patterns, i.e. $70.6 \%$ of the isolates.

Among the 49 clusters, we found 34 minor spoligotypes (including two to four isolates), and 15 major spoligotypes (five or more isolates). ST 66, 72, 385, 958, 1337, 1339, 1340, 1486, 1762, 1763, and 1935 are over-represented in French Guiana, i.e. they represents more than $50 \%$ of total number of isolates associated to these STs within the international spoligotype database SpolDB4. When spoligotypes were subtyped by VNTRs-excluding 14 isolates for which at least one VNTR failed - the number of cases per cluster decreased whereas the number of clusters slightly increased (52 clusters). Moreover, 17 spoligotype clusters on 49 were non-polymorphic for VNTR, i.e. all cases of a same spoligotype cluster shared the same VNTR profile. The main results of the molecular typing analysis are summarized on Table 1 and Figure 2. If we examine VNTR results of the five largest spoligotype clusters (including ten to forty-eight isolates), they were subdivided in 21 clusters (including two to twenty-four isolates) and 14 individual patterns (see Table 2 for details).

\section{Phylogeographic analysis}

Isolates of the $\mathrm{T}$ family predominated in our study $(32.6 \%$ of all isolates, $31.7 \%$ of all STs). The second major clade was the LAM family (23.3\% of all isolates, $27.5 \%$ of all STs) followed by the Haarlem family $(22.6 \%$ of all isolates, $10 \%$ of all STs), the EAI family (12.2\% of all isolates, $20 \%$ of all STs) and finally the X family (5.8\% of all isolates, $6.7 \%$ of all STs). Other families (M. africanum, Bei- 
Table I: Number and frequencies of isolates clustered by spoligotyping alone and further subtyped by VNTRs.

\begin{tabular}{lcc}
\hline & Isolates clustered by spoligotyping & Isolates clustered by spoligotyping and VNTR \\
\hline Total number of isolates studied & 344 & 339 \\
Number of clusters & 49 & 52 \\
Mean number of isolates per cluster & 5.57 & 3.90 \\
Number of clustered isolates (and \%) & $273(79.4 \%)$ & $203(59.9 \%)$ \\
Number of unclustered isolates (and \%) & $71(20.6 \%)$ & $127(37.5 \%)$ \\
\hline
\end{tabular}

jing, S) were under-represented in our setting. Figure 3 illustrates the complete cluster-graph, with all the 120 different spoligotypes censused in French Guiana through 1996 to 2003. The different clades are represented with different colours, and the size of nodes is proportional to the corresponding cluster size, i.e. the number of isolates per cluster. The cluster-graph reveals the likely ancestral genotype of each clade. Some edges were missing to construct the complete cluster-graph and were added, unless
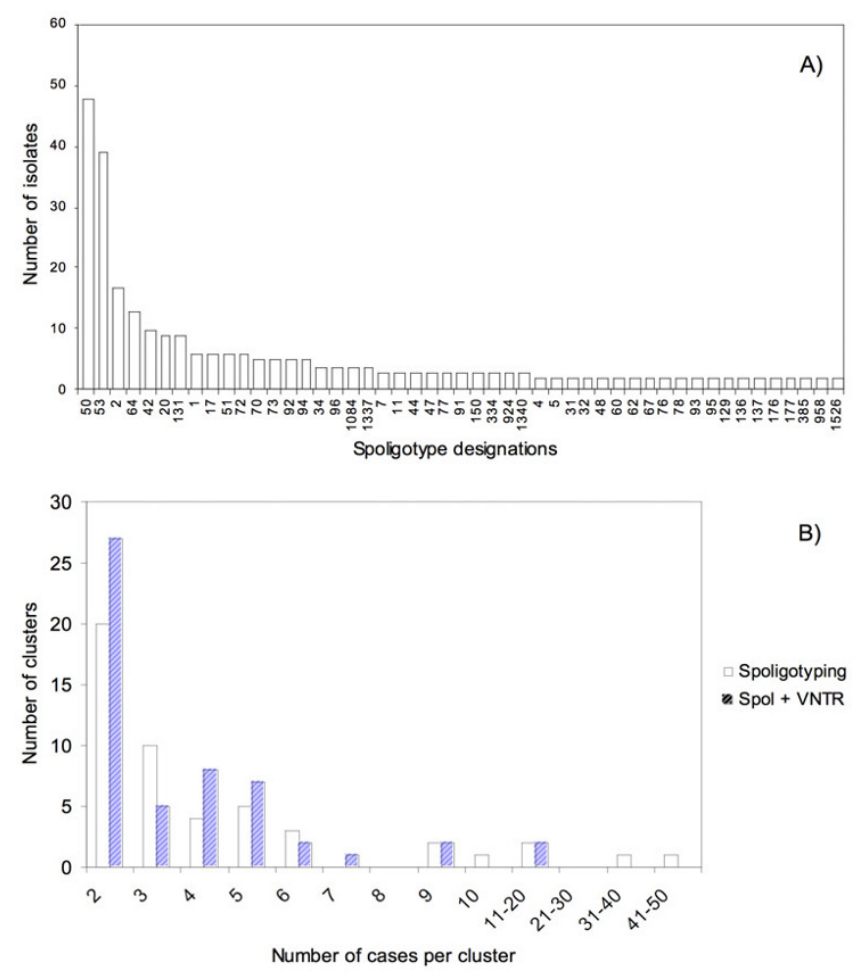

\section{Figure 2}

Synthetic histograms of the distribution of all isolates. (A) Frequency distribution of isolates associated to the 49 spoligotypes (STs) censused at least two times in French Guiana, southern America, over the 1996-2003 period; the most represented spoligotype is ST 50, with 48 isolates. (B) Frequency distributions of clusters ranked by cluster sizes, when clustered by spoligotyping technique (opened histograms), or when considering combined results of spoligotyping and VNTR (dashed histograms) (derived from table I). they were not censused in French Guiana during our survey (29 spoligotypes added, for example ST 119, putative ancestor of the X superfamily). In comparison, Figure 4 represents the minimum spanning tree produced using the BioNumerics software. Combined numerical analysis of spoligotyping and VNTR data for all 344 isolates underlined at least three well-defined branches (called A, C, D) emerging from a central cluster called $\mathrm{B}$. When considering each branch in detail, it can be shown that identified subgroups are most of the time not phylogenetically informative, i.e. isolates present in a branch are not family specific, except for branch D which is homogeneously constituted by LAM genotypes. Branch A contains genotypes from T, Haarlem, Beijing, LAM and X families; branch B shows genotypes from Haarlem, T, S and X families; and branch $\mathrm{C}$ contains genotypes from Haarlem, EAI, LAM, likely Haarlem and M. africanum.

\section{Estimation of transmission}

Applying the indices previously described to our French Guiana data set gives the following values: RTI $n=0.794$, RTI $n-1=0.651$, and TMI $=0.146$, with $n=344$ isolates, $n_{c}$ $=273$ isolates in cluster, $c=49$ clusters, $g=120$ spoligotypes and $v_{1}=82$ edges involving a 1 -step change. The number $v_{1}$ is equal to the number of genotypes $g$ in the data set minus the number of connected components, which is calculated using the cluster-graph (Figure 3 ). The RTI values here are very high compared to the same statistic computed on spoligotypes data (TMI), which means a lower recent transmission rate evaluated with TMI method. This difference is further explained in the discussion.

\section{Discussion \\ Molecular typing results}

Our genotypic results seems to show a low level of TB transmission as evidence through: (1) a high diversity of spoligotypes (120 individual patterns for 344 isolates), (2) a low mean cluster size (Table 1; Figure 2A) in spite of the high level of clustering $(79.4 \%$ of isolates included in clusters; Table 1), and (3) a high degree of unique STs $(59.2 \%)$, i.e. ST that cannot be related to a recent event of transmission. Moreover, the biggest clusters (48 and 39 isolates respectively; see Figure $2 \mathrm{~A}$ ) concern ubiquitous spoligotypes 50 and 53, widely spread on all the five con- 
Table 2: Detailed results of clustering for the five largest spoligotype clusters with combined spoligotyping - 5 VNTR loci genotyping.

\begin{tabular}{ccccc}
\hline Spoligotype & N & Number of different VNTR & Number of isolates/cluster & Individual patterns \\
\hline ST 53 & 39 & 14 & $6,5,5,4,4,3,2,2,2,2$ & 4 \\
ST 50 & 42 & 8 & $24,7,4,2,2$ & 3 \\
ST 42 & 10 & 7 & 3,2 & 5 \\
ST 64 & 13 & 4 & 9,2 & 2 \\
ST 02 & 15 & 2 & 11,4 & 0 \\
\hline
\end{tabular}

tinents (spreading index SI equal to 20.95 and 28.09 respectively [30]), and for which interpretation in terms of recent transmission remains difficult. However, we should keep in mind the suboptimal choice of the spoligotyping-VNTR genotyping scheme, which provided only a slight or no improvement compared to spoligotyping only. However, for practical reasons, and given the time frame covered (1996-2003), a full MIRU12 retrospective characterization of our sample could not be performed.

\section{Cluster-graph and minimum spanning tree}

Comparison between two clustering methods underlined major differences. The manual, parsimony-based, clustergraph method provides more coherent results, even if, in this study, we did not consider VNTRs to build the graph. The cluster-graph construction takes into account spoligotype evolution mechanisms, i.e. successive loss of one to many consecutive spacers by a deletion mechanism [31] whereas the algorithm used by BioNumerics takes into

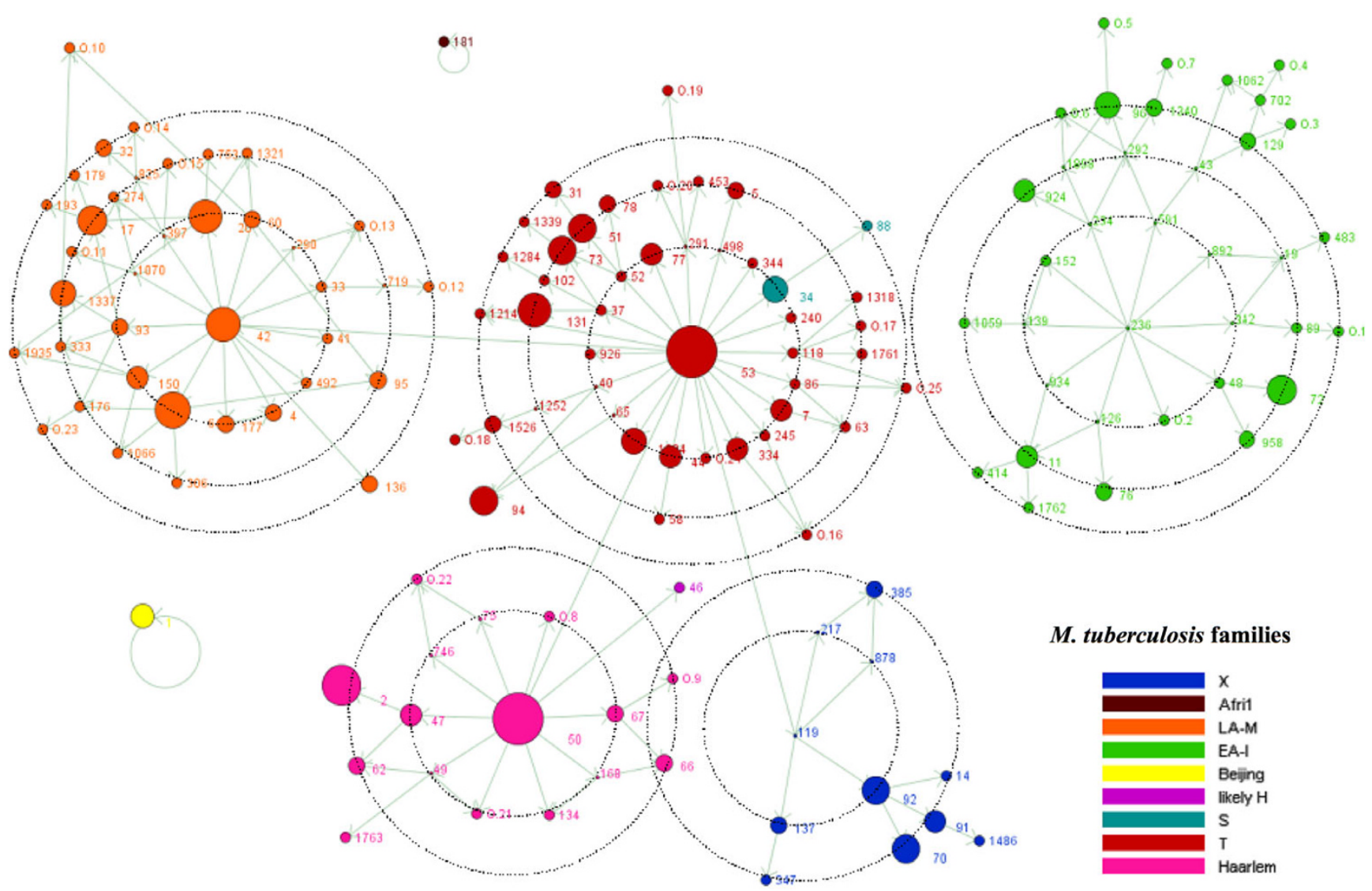

Figure 3

Cluster-graph drawn from spoligotyping data of 344 TB cases censused in French Guiana over the I996-2003 period. Each node represents a genotype, and arrows point in the direction of genotypes possibly derived by deletion in the marker locus. Vertex (or node) size indicates the size of the cluster. Twenty nine non-censused spoligotypes, which are hypothetical intermediates or ancestors of censused spoligotypes, were added and schematised as a point (e.g. STs I I9 and 236, putative ancestors of $\mathrm{X}$ clade and $\mathrm{EAl}$ clade, respectively). 


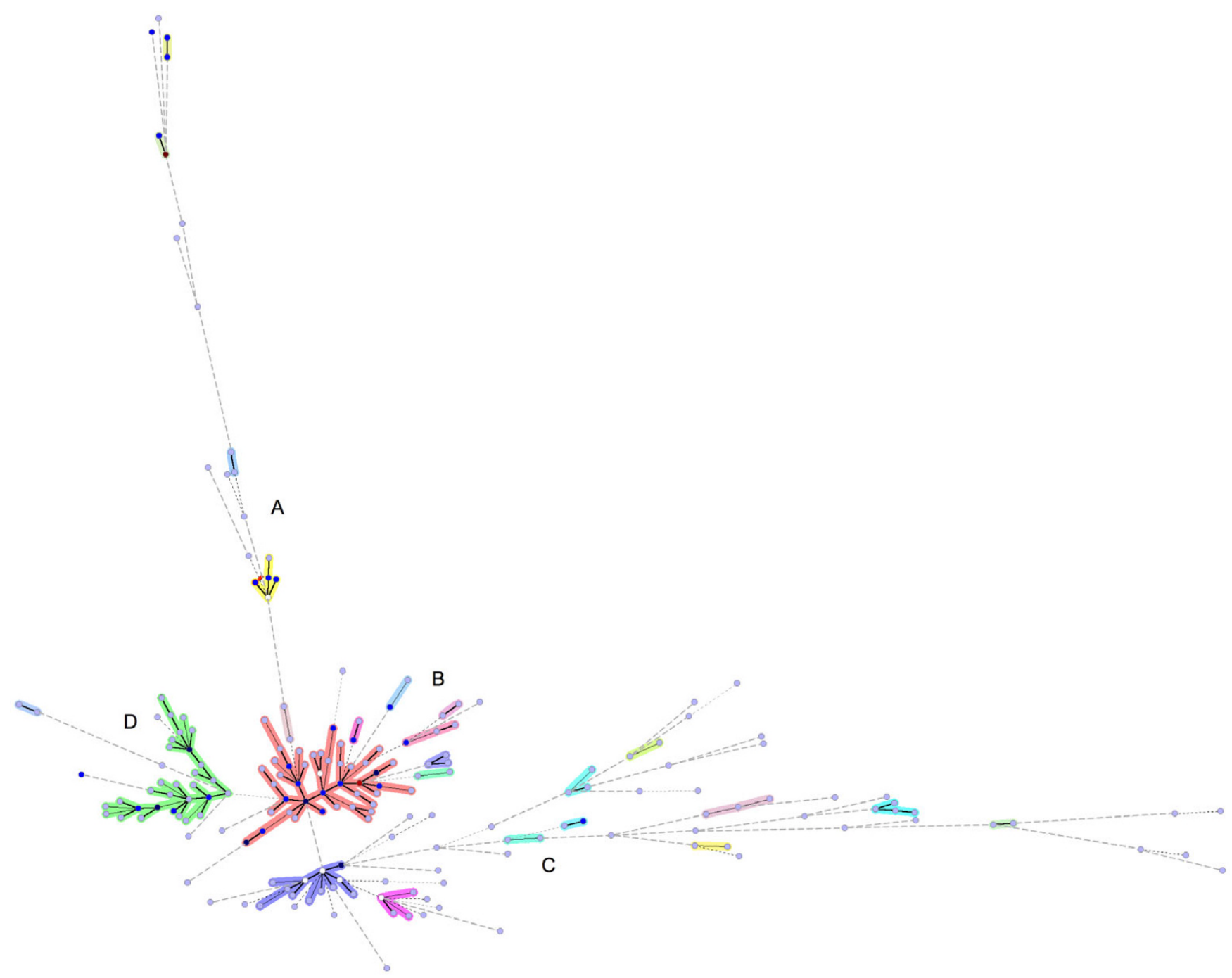

Figure 4

Minimum spanning tree. It was constructed using the I-Jaccard index and combining numerical analysis based on both spoligotyping and VNTR results. Options were chosen on BioNumerics software to allow the construction of hypothetical genotypes (which have not been censused in French Guiana), materialised on the tree as white circles. The four major branches are called A, B, C and D. Branch D is homogeneously constituted by LAM genotypes. Branch A contains genotypes from T, Haarlem, Beijing, LAM and $X$ families; branch $B$ contains genotypes from Haarlem, $T, S$ and $X$ families; branch $C$ shows genotypes from Haarlem, EAI, LAM, likely Haarlem and M. africanum.

account the maximal similarity between spoligotypes when considering the occurrence of the 43 spacers with respect to chronology from 1 to 43 . However such similarities do not systematically correspond to existing evolutionary mechanisms. Besides, it is well known that MST provides a distance-based analysis that may not be considered as a true phylogenetic tree. One example is spoligotypes 4, 2 and 1, which were phylogenetically related to branch A of the MST (Figure 4), based on identical absence of contiguous spacers 1 to 24, as illustrated in Figure 5 . In fact, those genotypes are from different families, i.e. Haarlem (classification rule: absence of spacer 31 and spacers 33-36), LAM (absence of spacers 21-24 and spacers 33-36) and Beijing clades (absence of spacers 1-34) respectively [32]. Finally, minimum spanning trees are more effective in case of exhaustive recruitments, in a very circumscribed and closed human community, which is not really the case in the present study.

\section{Phylogeography from spoligotyping}

Our results are coherent with migration and colonization history of human populations in French Guiana. The Haarlem clade (23\% of our strains) is considered to be a clade of European descent [33-35] and related spoligo- 


\begin{tabular}{|c|c|c|}
\hline ST & Clade & Binary description \\
\hline 004 & LAM9 & 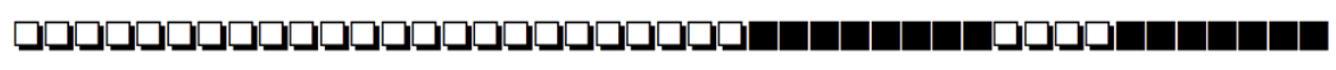 \\
\hline 002 & Haarlem2 & 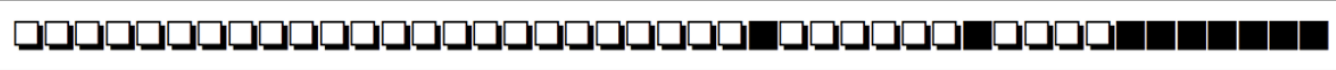 \\
\hline 001 & Beijing & " \\
\hline
\end{tabular}

Figure 5

Representative spoligotyping patterns of ST 01 , 02 and 04, and their associated clades. Dark squares indicate the presence of the spacer, and white squares indicate its absence (loss of spacers by a deletion mechanism). Unless they show some similarities (common absence of spacers I-24) the three genotypes ST 0I, 02 and 04 are from different families, i.e. Haarlem (absence of spacer 31 and spacers 33-36), LAM (absence of spacers 2I-24 and spacers 33-36) and Beijing clades (absence of spacers I-34) respectively. This illustrates that computation of distances on some types (for Minimum Spanning Tree construction) may be misleading.

types might have been introduced during previous colonization of the three Guiana (i.e. French Guiana, Suriname and Guyana) by migrants from France, Holland and Great Britain, respectively. Occurrence of $23 \%$ of spoligotypes belonging to the LAM clade (Latin-American and Mediterranean) might be best explained by population flows with neighbouring Latin-American countries, in particular Brazil, or by historical Lusitanian or Spanish settling. Concerning the EAI clade (East-African Indian; $12 \%$ of STs), the absence of many likely-ancestral STs was noticeable (e.g. ST 236, the putative EAI ancestor, and 7 out of 10 absent STs on the one-mutation circle illustrated in Figure $3)$, whereas distant genotypes were censused, presumably indicating a more recent expansion. The genotypes present in French Guiana might have been introduced by successive arrivals of Hakka settlers from Taiwan, Singapour, Hong-Kong, Vietnam and continental China since $1820 \quad$ http://www.tlfq.ulaval.ca/axl/amsudant/ guyanefr2.htm, and more recently, by settlers from the Lao-Hmong community which arrived in French Guyana in 1977 [36]. But there are obvious limitations to what can be achieved by spoligotyping, and the dominance of T strains in the current study (33\%) may (at best) demonstrate one of these. The $\mathrm{T}$ clade indeed remains an illdefined ubiquist family of $M$. tuberculosis found all over the world $[33,34]$, thus providing few phylogeographical information.

\section{Recent TB transmission in French Guiana}

The calculated TMI indicates a recent transmission rate of $14.6 \%$ on the $1996-2003$ period, instead of $79.4 \%$ and $65.1 \%$ of recent TB transmission calculated with RTI $n$ and RTI $n$-1 methods respectively. In a previous study [22], the rate of recent transmission was calculated to be $49.3 \%$ in French Guiana, using the RTI $n-1$ method based on the combined spoligotyping and VNTRs genotyping. The difference between our RTIn-1 result and the previous study is probably due to a different sampling and a smaller time frame of the present study (396 strains from 1994 to 2003 in [22] as compared to 344 isolates from 1996 to 2003 in the present study). Comparing RTI and TMI indices, we obtained a lower ongoing transmission rate with TMI method. The three methods are based on the same hypothesis about the potential epidemiological link between patients sharing the same $M$. tuberculosis strains. But the major weakness with RTI methods is their failure to account for strains diversity and mutation. The relationship between sample size $n$ and the number of observed genotypes $g$ is complex and depends on sampling, mutation, and population history, which were not integrated in previous RTI indices. In addition to being useful in visualizing data, cluster-graphs provide information about the abundance of particular genotypes in the sample, as well as partial information about possible evolutionary relationships, and can then be used as a way of understanding indices measuring the severity of outbreaks. The TMI result is the only one to be coherent with genotyping results described above (high genotypic diversity, small clusters, many orphans) which supposed a low level of recent $\mathrm{TB}$ transmission. The concomitant hypothesis is an endemic mode of TB transmission in French Guiana, with little local contamination occurring from person to person, but an overall persistence of the disease due to the large number of imported cases from countries with high TB incidence rates.

\section{New "transmission mutation index" method}

There are at least two biases that can occur when calculating TMI, and that may conduct to strictly opposite conclusions. The first possible bias is the emphasis of a single epidemic picture, whereas there are several parallel epidemics. For example, when considering two genotypes g1 and g2 differing by one single mutation step, the two isolates will be associated in the cluster-graph and thus related to a same epidemic, unless they potentially acquired the related genotypes independently by conver- 
gence, due to homoplasia. The infinite allele model, which underlies the TMI method, does not take into account such possibility, which can definitely occur.

The second possible bias is exactly the opposite, i.e. the emphasis of several epidemics, whereas there is only one. A sample is, by nature, incomplete. Thus, when isolates included in a cluster-graph are sampled from a population, some cases might be missing, which will induce a possible under-estimation of a cluster size, and/or overestimation of the number of different clusters. If one intermediate genotype is missing between two isolates, we will detect two epidemics unless there is a single transmission event.

A third bias can also be discussed here, considering the hypothesis we made that, when person A contaminates person $B$, their TB strains may differ at maximum by one mutation event. In fact, this hypothesis is likely to be true for spoligotypes, which have a low mutation rate. So, the possible bias due to points 1 and 2 are believed to be greater than this last one.

We must also notice that, because genotyping requires the availability of a viable isolate of $M$. tuberculosis, the population for which genotyping is performed must be a subset of all cases with positive cultures. In this study, 342 of 345 confirmed cases were spoligotyped. In turn, cases with positive cultures must be a subset of all cases (culture positive, negative, or not done). A previous study on the Ilede-Cayenne, French Guiana, showed that $61.7 \%$ of identified cases were confirmed by isolation and cellule culture of $M$. tuberculosis [15]. This must be taken into account when interpreting our results.

Last but not least, one should also pinpoint that no true analysis of patient demographical and medical data could be performed within the frame of this study. This is no doubt a strong limitation, since it is well known that molecular epidemiology performs well when collaboration between conventional and molecular epidemiology is achieved. The main reason relates to the difficulty to provide adequate human resources that would work for such a long period on the epidemiology of tuberculosis; however, even if a careful case-by-case analysis of the patient file data could not be assessed within the frame of the study, we fully assume that our results are a mirror of the epidemic situation of tuberculosis in French Guiana.

\section{Conclusion}

Our results confirmed that the use of cluster-graphs from spoligotyping data to derive a TMI index provides more sensitive results than previous RTI indices in order to measure the severity of outbreaks. It allowed us to show an endemic mode of TB transmission in French Guiana associated to low local transmission of the disease, with both resurgence of old spatially restricted genotypes (endemic to French Guiana), and an importation of new TB genotypes by migration of TB infected people from high-incidence countries. Endemic genotypes might also be due, to a lower extent, to local mutation events. Those results highlight the need for specific strategies of TB control in this region, considering those new epidemiological hypotheses.

This study also suggested that our methods choice in order to get a clear picture of the TB snapshot in a high prevalence setting such as French Guiana was indeed suboptimal. Genotyping of pathogens is a fast moving field that also requires long-term experience and stability of methods to provide golden standards in molecular characterization. A decade of IS6110-RFLP predominance, has now been efficiently challenged by the power of the Multi Locus VNTR analysis (MLVA) approach, which has proven to be useful for many pathogens. Meanwhile, the spoligotyping approach, once thought to be not discriminative enough, has revealed to be an excellent long-term choice in TB genotyping, not only because it is cheap and robust, but also because the underlying role of the DR (a member of the CRISPR family of repeats) is now better understood [37], and because updated databases are regularly maintained. An ultimate player in this game, i.e. SNP genotyping, could become the most useful tool during the next decade $[38,39]$. However, for the time-being, there is no emerging consensus on what is the best genotyping scheme to understand TB transmission based on molecular characterization of clinical isolates.

\section{Competing interests}

The author(s) declare that they have no competing interests.

\section{Authors' contributions}

VG conceived the study, completed the data analysis and prepared the final draft of the manuscript. CS participated to the data gathering and provided expertise in phylogeographical data analysis and manuscript writing. KB participated in experimental design and molecular typing. JFG helped participate in study design. NR supervised the identification and drug-susceptibility testing of tubercle bacilli, generated the molecular genetic data and participated in data analysis. All authors read the manuscript, participated in editing the manuscript and approved the final version.

\section{Availability and requirements}

At the time of the matching analysis, the updated spolDB4 version contained 31,642 patterns distributed into 2,393 shared types in 114 countries (an online version of this 
database is now available at http://www.pasteur-guade loupe.fr:8081/SITVITDemo/).

The genotypes present in French Guiana might have been introduced by successive arrivals of Hakka settlers from Taiwan, Singapour, Hong-Kong, Vietnam and continental China since 1820 http://www.tlfq.ulaval.ca/axl/amsu dant/guyanefr2.htm, and more recently, by settlers from the Lao-Hmong community which arrived in French Guyana in 1977.

\section{Acknowledgements}

VG acknowledges the French Ministry of Higher Education and Research for providing a Ph.D. fellowship. The research done at the Pasteur Institute of Guadeloupe benefited through a Ph.D. fellowship awarded to KB (partially financed by the European Social Funds provided through the Regional Council of Guadeloupe), and research grants awarded to NR by the International Network of the Pasteur Institutes, Direction of International Affairs, Institut Pasteur, Paris. JFG acknowledges the Institut de Recherche pour le Développement and the Centre National de la Recherche Scientifique for their financial, administrative and technical support. Thanks are due to Dr. Andrew Francis for its help on applying the method of clustergraph to our data set and for very useful comments, and to Drs. P. Duval and B. Maubert (Pasteur Institute of French Guiana) for providing samples. We are also grateful to Drs. N. Quintard, B. Cottrelle, D. Louvel, B. Carme, J. Thonnon, R. Pradineau, G. Guillot, and A.-M. Bourbigot in French Guiana for their help during epidemiological investigations around clustered cases.

\section{References}

I. Hopewell PC: Tuberculosis control: how the world has changed since 1990. Bulletin of the World Health Organization 2002, 80(6): 427 .

2. Barnes PF, El-Hajj H, Preston-Martin S, Cave MD, Jones BE, Otaya M, Pogoda J, Eisenach KD: Transmission of tuberculosis among the urban homeless. JAMA 1996, 275:305-307.

3. Lukacs J, Tubak V, Mester J, David S, Bartfai Z, Kubica T, Niemann S, Somoskovi A: Conventional and Molecular Epidemiology of Tuberculosis in Homeless Patients in Budapest, Hungary. J Clin Microbiol 2004, 42(I 2):593I-5934.

4. Reichler MR, Bur S, Reves R, Mangura B, Thompson V, Ford J, Castro $K G$ : Results of testing for human immunodeficiency virus infection among recent contacts of infectious tuberculosis cases in the United States. Int J Tuberc Lung Dis 2003, 7( 2 Suppl 3):S47I-8.

5. Schaaf HS, Krook S, Hollemans DW, Warren RM, Donald PR, Hesseling AC: Recurrent culture-confirmed tuberculosis in human immunodeficiency virus-infected children. Pediatr Infect Dis / 2005, 24(8):685-69|

6. Monot M, Honore N, Garnier T, Araoz R, Coppée JY, Lacroix C, Sow $S$, Spencer JS, Truman RW, Williams DL, Gelber R, Virmond M, Flageul B, Cho S-N, Ji B, Paniz-Mondolfi A, Convit J, Young S, Fine PE, Rasolofo V, Brennan PJ, Cole ST: On the origin of leprosy. Science 2005, 308(5724): 1040-1042.

7. Small $P$, Behr M: Molecular fingerprinting of Mycobacterium tuberculosis: how can it help the clinician? Clinical Infectious Diseases 1997, 25:806-810.

8. Crawford JT, Braden CR, Schable BA, Onorato IM: National Tuberculosis Genotyping and Surveillance Network: design and methods. Emerg Infect Dis 2002, 8(I I): I | 92-1 I96.

9. Moström P, Gordon M, Sola C, Ridell M, Rastogi N: Methods used in the molecular epidemiology of tuberculosis. Clin Microbiol Infect 2002, 8(I I):694-704.

10. Alland D, Kalkut G, Moss A, McAdam R, Hahn JA, Bosworth W, Drucker E, Bloom BR: Transmission of tuberculosis in New York City. An analysis by DNA fingerprinting and conventional epidemiologic methods. N Engl J Med 1994, 330(24): 1710-1716.
II. Small PM, Hopewell PC, Singh SP, Paz A, Parsonnet J, Ruston DC, Schecter GF, Daley CL, Schoolnik GK: The epidemiology of tuberculosis in San Francisco: A population-based study using conventional and molecular methods. N EnglJ Med I994, 330:1703-1709.

12. Seidler A, Nienhaus A, Diel R: The transmission of tuberculosis in the light of new molecular biological approaches. Occup Environ Med 2004, 6 I (2):96-102.

13. Kulaga S, Behr MA, Schwartzman K: Genetic fingerprinting in the study of tuberculosis transmission. CMAJ 1999, 161(9): I I65-II69.

14. Tanaka MM, Francis AR: Methods of quantifying and visualising outbreaks of tuberculosis using genotypic information. Infect Genet Evol 2005, 5(I):35-43.

15. Guernier V, Guegan JF, Deparis X: An evaluation of the actual incidence of tuberculosis in French Guiana using a capturerecapture model. Microbes Infect 2006, 8(3):72I-727.

16. Filliol I, Ferdinand S, Negroni L, Sola C, Rastogi N: Molecular typing of Mycobacterium tuberculosis based on variable number of tandem DNA repeats used alone and in association with spoligotyping. J Clin Microbiol 2000, 38(7):2520-2524.

17. Sola C, Filliol I, Legrand E, Lesjean S, Locht C, Supply P, Rastogi N: Genotyping of the Mycobacterium tuberculosis complex using MIRUs: association with VNTR and spoligotyping for molecular epidemiology and evolutionary genetics. Infect Genet Evol 2003, 3(2): I25-133.

18. Supply P, Allix C, Lesjean S, Cardoso-Oelemann M, Rüsch-Gerdes S, Willery E, Savine E, de Haas P, van Deutekom H, Roring S, Bifani P, Kurepina N, Kreiswirth B, Sola C, Rastogi N, Vatin V, Gutierrez MC, Fauville M, Niemann S, Skuce R, Kremer K, Locht C, van Soolingen D: Proposal for standardization of optimized mycobacterial interspersed repetitive unit-variable-number tandem repeat typing of Mycobacterium tuberculosis. J Clin Microbiol 2006, 44(I 2):4498-45 I0.

19. van Soolingen D, de Haas PE, Hermans PWM, van Embden JDA: DNA fingerprinting of Mycobacterium tuberculosis. Methods Enzymol 1994, 235:196-205.

20. Kamerbeek J, Schouls L, Kolk A, van Agterveld M, van Soolingen D, Kuijper S, Bunschoten A, Molhuizen H, Shaw R, Goyal M, van Embden J: Simultaneous detection and strain differentiation of Mycobacterium tuberculosis for diagnosis and epidemiology. J Clin Microbiol 1997, 35:907-914.

21. Goguet de la Salmoniere YO, Li HM, Torrea G, Bunschoten A, van Embden J, Gicquel B: Evaluation of spoligotyping in a study of the transmission of Mycobacterium tuberculosis. J Clin Microbiol 1997, 35:2210-2214.

22. Brudey K, Filliol I, Ferdinand S, Guernier V, Duval P, Maubert P, Sola $S$, Rastogi N: Long-term population-based genotyping study of Mycobacterium tuberculosis complex isolates in the French Departments of the Americas. J Clin Microbiol 2006, 44(I): $|83-19|$.

23. Frothingham R, Meeker-O'Connell WA: Genetic diversity in the Mycobacterium tuberculosis complex based on variable numbers of tandem DNA repeats. Microbiology 1998, I44: I I 89- I I 96.

24. Brudey K, Driscoll JR, Rigouts L, Prodinger WM, Gori A, Al-Hajoj SA, Allix C, Aristimuño L, Arora J, Baumanis V, Binder L, Cafrune P, Cataldi A, Cheong S, Diel R, Ellermeier C, Evans JT, Fauville-Dufaux M, Ferdinand S, Garcia de Viedma D, Garzelli C, Gazzola L, Gomes HM, Guttierez MC, Hawkey PM, van Helden PD, Kadival GV, Kreiswirth BN, Kremer K, Kubin M, Kulkarni SP, Liens B, Lillebaek T, Ho ML, Martin C, Martin C, Mokrousov I, Narvskaïa O, Ngeow YF, Naumann L, Niemann S, Parwati I, Rahim Z, Rasolofo-Razanamparany V, Rasolonavalona T, Rossetti ML, Rüsch-Gerdes S, Sajduda A, Samper S, Shemyakin IG, Singh UB, Somoskovi A, Skuce RA, van Soolingen D, Streicher EM, Suffys PN, Tortoli E, Tracevska T, Vincent V, Victor TC, Warren RM, Yap SF, Zaman K, Portaels F, Rastogi N, Sola C: Mycobacterium tuberculosis complex genetic diversity: mining the fourth international spoligotyping database (SpolDB4) for classification, population genetics, and epidemiology. BMC Microbiol 2006, 6:23.

25. Enright AJ, Ouzounis CA: BioLayout - an automatic graph layout algorithm for similarity visualization. Bioinformatics $200 \mathrm{I}$, I7(9):853-854.

26. Goldovsky L, Cases I, Enright AJ, Ouzounis CA: BioLayoutJava: versatile network visualisation of structural and functional relationships. Applied Bioinformatics 2005, 4(I):7I-74. 
27. Glynn JR, Vynnycky E, Fine PE: Influence of sampling on estimates of clustering and recent transmission of Mycobacterium tuberculosis derived from DNA fingerprinting techniques. Am J Epidemiol 1999, 149:366-37।.

28. Murray M: Sampling bias in the molecular epidemiology of tuberculosis. Emerg Infect Dis 2002, 8(4):363-369.

29. Rosenberg NA, Tsolaki AG, Tanaka MM: Estimating change rates of genetic markers using serial samples: applications to the transposon IS 6110 in Mycobacterium tuberculosis. Theor Popul Biol 2003, 63(4):347-363.

30. Filliol I, Driscoll JR, van Soolingen D, Kreiswirth BN, Kremer K, Valétudie G, Dang DA, Barlow R, Banerjee D, Bifani PJ, Brudey K, Catald A, Cooksey RC, Cousins DV, Dale JW, Dellagostin OA, Drobniewski F, Engelmann G, Ferdinand S, Gascoyne-Binzi D, Gordon M, Gutierrez MC, Haas WH, Heersma H, Kassa-Kelembho E, Ho ML, Makristathis A, Mammina C, Martin G, Moström P, Mokrousov I, Narbonne V, Narvskaya O, Nastasi A, Niobe-Eyangoh SN, Pape JW, RasolofoRazanamparany V, Ridell M, Rossetti ML, Stauffer F, Suffys PN, Takiff $\mathrm{H}$, Texier-Maugein J, Vincent $\mathrm{V}$, de Waard JH, Sola C, Rastogi $\mathrm{N}$ : Snapshot of moving and expanding clones of Mycobacterium tuberculosis and their global distribution assessed by spoligotyping in an international study. J Clin Microbiol 2003, 4I(5): 1963-1970

31. Warren RM, Streicher EM, Sampson SL, van der Spuy GD, Richardson M, Nguyen D, Behr MA, Victor TC, van Helden PD: Microevolution of the Direct Repeat region of Mycobacterium tuberculosis: implications for interpretation of spoligotyping data. J Clin Microbiol 2002, 40(I 2):4457-4465.

32. Filliol I, Driscoll JR, Van Soolingen D, Kreiswirth BN, Kremer K, Valétudie G, Anh DD, Barlow R, Banerjee D, Bifani PJ, Brudey K, Cataldi A, Cooksey RC, Cousins DV, Dale JW, Dellagostin OA, Drobniewski F, Engelmann G, Ferdinand S, Gascoyne-Binzi D, Gordon M, Gutierrez MC, Haas WH, Heersma H, Källenius G, Kassa-Kelembho E, Koivula T, Ly HM, Makristathis A, Mammina C, Martin G, Moström P, Mokrousov I, Narbonne V, Narvskaya O, Nastasi A, Niobe-Eyangoh SN, Pape JW, Rasolofo-Razanamparany V, Ridell M, Rossetti ML Stauffer F, Suffys PN, Takiff H, Texier-Maugein J, Vincent V, De Waard $\mathrm{JH}$, Sola C, Rastogi N: Global distribution of Mycobacterium tuberculosis spoligotypes. Emerg Infect Dis 2002, 8: I347-I349.

33. Singh UB, Suresh N, Bhanu NV, Arora J, Pant H, Sinha S, Aggarwal RC, Singh S, Pande JN, Sola C, Rastogi N, Seth P: Predominant tuberculosis spoligotypes, Delhi, India. Emerg Infect Dis 2004, 10:1138-1142.

34. Sola C, Filliol I, Guttierez C, Mokrousov I, Vincent V, Rastogi N: Spoligotype database of Mycobacterium tuberculosis: biogeographical distribution of shared types and epidemiological and phylogenetic perspectives. Emerg Infect Dis 200I, 7:390-396.

35. Kremer K, van Soolingen D, Frothingham R, Haas WH, Hermans PW, Martín C, Palittapongarnpim P, Plikaytis BB, Riley LW, Yakrus MA, Musser JM, van Embden JD: Comparison of methods based on different molecular epidemiological markers for typing of Mycobacterium tuberculosis complex strains: interlaboratory study of discriminatory power and reproducibility. J Clin Microbiol 1999, 37:2607-26I8.

36. Barret J, (Dir): Atlas illustré de la Guyane 2nd edition. Limoges: IRD; 200I.

37. Barrangou R, Fremaux C, Deveau H, Richards M, Boyaval P, Moineau $S$, Romero DA, Horvath P: CRISPR provides acquired resistance against viruses in prokaryotes. Science 2007, 315(58|9): I709-|7|2.

38. Filliol I, Motiwala AS, Cavatore M, Qi W, Hazbón MH, Bobadilla del Valle M, Fyfe J, García-García L, Rastogi N, Sola C, Zozio T, Guerrero MI, León CI, Crabtree J, Angiuoli S, Eisenach KD, Durmaz R, Joloba $M L$, Rendón A, Sifuentes-Osornio J, Ponce de León A, Cave MD, Fleischmann R, Whittam TS, Alland D: Global phylogeny of Mycobacterium tuberculosis based on single nucleotide polymorphism (SNP) analysis: insights into tuberculosis evolution, phylogenetic accuracy of other DNA fingerprinting systems, and recommendations for a minimal standard SNP set. J Bacteriol 2006, I 88(2):759-772

39. Gutacker MM, Mathema B, Soini H, Shashkina E, Kreiswirth BN, Graviss EA, Musser JM: Single-nucleotide polymorphism-based population genetic analysis of Mycobacterium tuberculosis strains from 4 geographic sites. J Infect Dis 2006, 193(I): $121-128$

\section{Pre-publication history}

The pre-publication history for this paper can be accessed here:

http://www.biomedcentral.com/1471-2334/8/46/prepub
Publish with Biomed Central and every scientist can read your work free of charge

"BioMed Central will be the most significant development for disseminating the results of biomedical research in our lifetime. "

Sir Paul Nurse, Cancer Research UK

Your research papers will be:

- available free of charge to the entire biomedical community

- peer reviewed and published immediately upon acceptance

- cited in PubMed and archived on PubMed Central

- yours - you keep the copyright 